\title{
Form Factor of Eucalyptus Saligna Species in a Commercial Forest Plantation
}

\section{Factor de Forma de la Especie Eucalyptus Saligna en una Plantación Forestal Comercial}

Memories II International Congress Forests and Agroforestry for the 21st Century

Corresponding Author:

M. Guallpa

miguel.guallpa@espoch.edu.ec

Published: 21 January 2021

Production and Hosting by Knowledge $E$

(c) N. Lara et al. This article is distributed under the terms of the Creative Commons

Attribution License, which permits unrestricted use and redistribution provided that the original author and source are credited.

\author{
N. Lara1, M. Guallpa1, F. Acosta1, and Á. Barahona² \\ ${ }^{1}$ Carrera de Ingeniería Forestal, Facultad de Recursos Naturales, Escuela Superior Politécnica \\ de Chimborazo, Riobamba, Ecuador \\ ${ }^{2}$ Novopan S.A., QUITO, Pichincha, Ecuador
}

\section{Abstract}

The present investigation proposes to determine the form factor of the species Eucalyptus saligna in a commercial forest plantation of the Tambillo bajo sector, of the Colta canton, Chimborazo province. For this purpose, 100 individual specimens with straight shafts and without bifurcations were taken as a sample, and each individual's commercial and total height, diameter at breast height, and diameters along the stem were measured, to determine the real volume of the wood using the Smalian and Newton formulas, the graphical method, and with a form factor of 0.70 . Based on the measured data, a comparative analysis of the calculation of real volume by the method used was performed; the results obtained showed significant differences between calculation methods. In addition, five potential volume equations that presented the highest adjusted coefficient of determination and the lowest mean square error were selected. To verify the commercial volume calculated with the estimated form factors against the volume equations, the commercial volume was recalculated based on the form factors and equations found. The calculation of the commercial volume by the Smalian formula is taken as the exact value, and the other methods are taken as approximated values.

Keywords: dasometry, form factor, forest plantation, volumen equations, commercial volume.

\section{Resumen}

La presente investigación propone: determinar el factor de forma de la especie Eucalyptus saligna en una plantación forestal comercial; para lo cual se toma como muestra 100 individuos con fustes rectos y sin bifurcaciones, a cada individuo se midió la altura comercial y total, diámetro a la altura del pecho y diámetros a lo largo del fuste, para determinar el volumen real de madera utilizando la fórmula de Smalian, Newton, método gráfico y con un factor de forma de 0,70. En base a los datos medidos, se realiza un análisis comparativo del cálculo de volumen real por método empleado, los resultados obtenidos mostraron diferencias significativas entre métodos de cálculo empleados. Además, se seleccionó 5 ecuaciones de volumen potenciales los cuales presentaron mayor coeficiente de determinación ajustado y menor error cuadrático medio. Para la verificación del volumen comercial calculado con los factores de forma estimados frente a las ecuaciones de volumen se vuelve a calcular el volumen comercial a partir de los factores de forma y ecuaciones encontrados, se toma como valor exacto el cálculo de volumen comercial por la fórmula de Smalian y como valor de aproximación los demás métodos. 


\section{Introducción}

El factor de forma es una característica propia para cada especie en el cálculo del volumen comercial, debido a la disminución del diámetro, desde la base del árbol conforme aumenta la altura, lo que confirma que es importante en el proceso de datos usar factores de forma propios para cada familia y si fuera posible llegar a factores para cada especie. Por tal razón la Dirección de Producción Forestal establece la metodología para la determinación de factores de forma para especies forestales, el objetivo es establecer normas técnicas mediante las cuales se regulen la elaboración, aprobación, ejecución de los programas de corta y las licencias de aprovechamiento forestal [1-3].

Para el cálculo del volumen comercial, ha sido necesario el uso de modelos matemáticos cuya importancia radica en la optimización de los recursos económicos y tiempo. Mediante esta estimación se relaciona la variable dependiente (volumen comercial) con una o varias variables independientes de fácil medición como por ejemplo diámetro a la altura del pecho, altura total, altura comercial, área basal, siendo esta la manera que se puede materializar de modo más eficiente y sirva de insumo para la valoración más adecuada del volumen de madera en pie permitiendo la organización de su producción a nivel de fábricas [4].

Por lo tanto, el uso de modelos matemáticos juega un rol importante al momento verificar el volumen calculado con los factores de forma estimados, ya que genera información cuantitativa sobre la disponibilidad, ordenación con fines de comercialización y aprovechamiento de la especie en estudio [5]. Con el propósito de cumplir con los fines indicados, se planteó determinar el factor de forma de la especie Eucalyptus saligna en una plantación forestal comercial con la finalidad de precisar la cuantificación del volumen comercial.

\section{Materiales y Métodos}

La presente investigación se desarrolló en una plantación forestal de 17,6 hectáreas ubicadas en el sector Tambillo Bajo, ubicada a 22 km del cantón Colta, Provincia de Chimborazo (Tabla 1). La temperatura media anual es de $13,5^{\circ} \mathrm{C}$, con una precipitación media anual de $1186,42 \mathrm{~mm}$, la humedad relativa anual máxima de 61,26\% y mínima $51,29 \%$ [6]. Se encuentra en una formación vegetal bosque siempre verde montano del Norte de la cordillera oriental de los Andes [7].

\section{Table 1}

Ubicación geográfica del área de estudio.

\begin{tabular}{|l|l|l|l}
\hline Datum & Latitud & Longitud & Altitud \\
\hline $\begin{array}{l}\text { Coordenadas Proyectadas UTM Zona 17S, } \\
\text { DATUM WGS 84 }\end{array}$ & 721653 & 9792934 & 2076 m.s.n.m. \\
\hline
\end{tabular}


En la investigación se aplicaron los métodos: Documental, exploratoria, descriptiva y de campo [8]. El muestreo de las variables cuantitativas tuvo lugar el segundo semestre del año 2018.

\subsection{Procedimiento para el análisis comparativo del cálculo de vol- umen real por secciones para la estimación del factor de forma de la especie Eucalyptus saligna}

Delimitación del área de estudio: Mediante la visita y reconocimiento de la plantación comercial forestal ubicada en el sector Tambillo Bajo, cantón Colta, provincia de Chimborazo, se identificó sus límites, marco de plantación, edad de la plantación a fin de levantar su mapa y determinar la superficie total a ser aprovechada.

Selección de árboles: Se seleccionó 100 árboles representativos para el muestreo, los árboles sin deformaciones, fuste recto y que no presenten bifurcaciones fueron objeto del presente estudio.

Medición del DAP: Se tomó el diámetro a $130 \mathrm{~cm}$ del suelo (DAP) y se marcó con pintura de color rojo en donde se realizó la medición utilizando una forcípula.

Tumba de árboles seleccionados: La tumba de los árboles seleccionados se realizó basado en los lineamientos definidos por la empresa NOVOPAN S.A. En el proceso de tumba se utilizó dos motosierras que apeaban y cuadraban la base de los árboles.

Medición de los diámetros en cada sección: Se realizó la medición de las secciones del árbol apeado, primero se midió el tocón desde la altura del suelo hasta el corte la primera troza a la cuál denominamos A1., la segunda medición se realizó desde el corte hasta la sección que corresponde al corte corrección en la primera troza a la cual denominamos A2., desde A2., se realizaron las mediciones de seccionamiento a cada 1 metro iniciando a partir de la primera marca (D1) hasta llegar al diámetro mínimo comercial de $5 \mathrm{~cm}$ (Dc), en cada punto de seccionamiento se efectuó marcas de pintura. Luego se realizó el seccionamiento con marcas de pintura y se procedió a medir con forcípula los diámetros de cada sección.

Medición de la altura total: Una vez cortado y realizado las marcas respectivas al árbol, se procedió a medir la longitud desde el D1 hasta llegar al diámetro mínimo de corta de $5 \mathrm{~cm}$, su altura total corresponde a la sumatoria de la longitud de todas las secciones más la punta del mismo.

Formulario de campo y elaboración de una base de datos en Excel: Las mediciones efectuadas sobre los árboles apeados en campo se llenaron en el formulario de campo y se crea una base de datos en Excel que incluye el cálculo del volumen real de cada unidad experimental en estudio $[9,10]$.

Los datos que se obtienen provienen de operaciones matemáticas con las variables que se encuentran en la hoja de ingreso de datos. La altura comercial de cada individuo resulta de la sumatoria de la longitud de todas las secciones hasta la sección que tiene $5 \mathrm{~cm}$ de diámetro. 
La fórmula para el sólido de referencia para cada árbol es la siguiente:

$$
\text { Vol. cilindro }=\left(\frac{D A P}{2}\right)^{2} * \pi * h c,
$$

donde: Vol. cilindro: Volumen del solido de referencia (cilindro); DAP: Diámetro a la altura del pecho; $\pi$ : 3,1416; $h c$ : Altura comercial de cada individuo.

Para el cálculo de volumen real para cada sección se utilizó las fórmulas de Smalian y Newton:

Fórmula de Smalian; Aparece en 1806. Se deben evaluar las áreas de las secciones extremas C1 y C2 y su longitud. El volumen real se valora mediante la expresión [11]:

$$
\mid V_{S}=\frac{\pi}{4}\left[\frac{C_{1}^{2}+C_{2}^{2}}{2}\right] L,
$$

donde: $V_{s}$ : Es el volumen por el cálculo de Smalian; $\pi$ : Es Pi (3,1415.); $C_{1}^{2}$ : es el área de sección fustal al inicio de la troza; $C_{2}^{2}$ : es el área de sección fustal al final de la troza; $\mathrm{L}$ : es la longitud del fuste o troza.

Fórmula de Newton; En 1940 se generaliza la fórmula propuesta por Then. Se aplica a un mayor número de cuerpos geométricos. Para la estimación del volumen real se emplea [11]:

$$
V_{s}=\frac{\pi}{4}\left[\frac{C_{1}^{2}+4 C_{m}^{2}+C_{2}^{2}}{6}\right] L,
$$

donde: $V_{s}$ : Es el volumen por el cálculo de Smalian; $\pi$ : Es Pi $(3,1415 \ldots) ; C_{1}^{2}$ : Es el área de sección fustal al inicio de la troza; $C_{m}^{2}$ : Es el área de sección media fustal de la troza; $C_{2}^{2}$ : Es el área de sección fustal al final de la troza; $L$ : Es la longitud del fuste o troza.

El volumen real total de cada individuo resulta de la sumatoria del volumen real encontrado en cada sección.

El factor de forma de cada árbol resulta de dividir el volumen real total de ese árbol para el volumen del cilindro del mismo.

Cálculo del volumen real por el método gráfico mediante el programa SolidWorks: Mediante el software de diseño asistido por computador SolidWorks versión 2017, se modelizó cada fuste para cada árbol individualmente.

Cálculo del volumen del testigo: Para el cálculo del volumen real del testigo se establece el valor de 0,70 como factor de forma, presente en el artículo 10 del acuerdo ministerial No. 327 emitido por el Ministerio de Agricultura y Ganadería [12].

Finalmente, los factores de forma que se presentaron en este estudio resultaron del promedio de los 100 factores de forma encontrados por cada método de cubicación.

Análisis estadísticos y comparaciones: Los datos del volumen individual fueron transformados a logaritmo natural con el fin de mejorar el desempeño de las medidas de distancia composicional, además se realizaron los siguientes análisis estadístico en el programa SPSS [13]. Los cuales fueron: 
Pruebas de Normalidad: Se realizó el gráfico Q-Q plot para evaluar el grado de ajuste del conjunto de datos en estudio a una distribución normal. La prueba de KolmogorovSmirnov para medir el grado de concordancia existente entre la distribución de los valores del volumen individual y una distribución normal, es decir si las observaciones podrían razonablemente proceder de la distribución normal.

Estadísticos descriptivos: Se analizaron las medidas resumen para comparar conjuntos de datos cuantitativos y para presentar los resultados del estudio.

ANOVA: Se efectuó el análisis de varianza al volumen real y a cuatro tipos de ecuaciones para volumen con los coeficientes de correlación lineal de Pearson (R) más altos, para determinar si realmente existe una relación de dependencia entre las variables objeto de estudio. Se complementó con la prueba de Tukey para realizar todas las comparaciones entre las medias del volumen comercial $\mathrm{m}^{3}$ de los distintos métodos de cálculos empleados en este estudio.

Los datos del factor de forma provienen de una estadística no paramétrica, se rigen a los siguientes análisis estadísticos en el programa SPSS:

Pruebas no paramétricas: Se efectuó la prueba de Kruskal-Wallis a los factores de forma encontrados por las fórmulas de Smalian, Newton, el método gráfico y testigo, debido a que el conjunto de datos no presenta una distribución normal.

Gráfico de medianas: Se realizó un gráfico de cajas para representar el valor de las medianas de los factores de forma encontrados dentro de este estudio.

\subsection{Procedimiento para verificar el volumen calculado con los fac- tores de forma estimados mediante ecuaciones de volumen de una y doble entrada para la especie en estudio}

Obtención de los modelos a utilizar en los ajustes: Se realizó una tabla de correlaciones posibles en el programa estadístico Excel combinando las variables medidas, con el fin de encontrar las ecuaciones de volumen potenciales y eliminar aquellas que por baja correlación no justificaban su inserción [14].

Selección del modelo: Se eligió cinco ecuaciones de volumen potenciales y para ello se utilizó el ajuste de correlación (R) entre la variable dependiente-independiente. Seleccionando aquel modelo que presentó mayor ajuste de correlación (R) entre todos los modelos [14].

Regresiones: Se ejecutó una regresión lineal en el programa estadístico SPSS a cada ecuación de volumen potencial ajustando a la relación matemática entre el volumen real encontrado con la fórmula de Smalian (variable dependiente) y las variables en estudio (DAP, altura) para determinar una confiabilidad aceptable cumpliendo con los supuestos de linealidad, normalidad y auto correlación.

Presentación de modelos matemáticos potenciales: Se presentan los modelos matemáticos con los valores del coeficiente de determinación y el valor $F$ critico encontrados en cada una de ellas [15, 16].

Análisis comparativo estadístico: Una vez encontrada las cinco ecuaciones de volumen potenciales y los factores de forma por los diferentes métodos de cubicación 
Smalian, Newton, método gráfico y testigo, se procedió a encontrar el volumen comercial a partir del diámetro a la altura del pecho y la altura comercial de los 100 árboles en estudio para encontrar su media y error relativo.

\section{Resultados y Discusión}

\subsection{Análisis comparativo del cálculo de volumen por secciones para la estimación del factor de forma de la especie Eucalyptus saligna}

En el Análisis de la Varianza (ANOVA) de la muestra del volumen real en $\mathrm{m}^{3}$ (Tabla 2), dado que el $p$-valor es menor que 0,05, se acepta la hipótesis que una o varias medias del método de cálculo para el volumen individual empleado en este estudio poseen diferencias entre sus medias.

\section{Table 2}

Análisis de la Varianza (ANOVA) de la variable volumen real.

\begin{tabular}{l|l|l|l|l|l|}
\hline F.V. & S.C. & G.L. & C.M & F & Sig. \\
\hline Inter-grupos & 6,823 & 3 & 2,274 & 7,483 & $0,000^{* * *}$ \\
\hline Intra-grupos & 120,353 & 396 & 0,304 & & \\
\hline Total & 127,176 & 399 & & & \\
\hline
\end{tabular}

F.V.: Fuente de variación; S.C.: Suma de Cuadrados; G.L.: Grados de libertad; C.M.: Cuadrado Medio; F: Fisher; Sig: Significancia.

En el análisis comparativo entre método de cálculo Smalian, Newton, método gráfico y testigo para el cálculo del volumen real por el test de Tukey con un intervalo de confianza al 95\%, se ubicó en el subconjunto ' 1 ' al cálculo por F. Smalian, Newton y método gráfico con medias de 0,631; 0,633; $0,720 \mathrm{~m}^{3}$ respectivamente, los cuales no tienen diferencias significativas y corrobora con el estudio en el cual toma los datos dasométricos de árboles apeados medidos los diámetros con intervalos de un metro, empezando en el tocón (a $20 \mathrm{~cm}$ del suelo) y el diámetro a la altura del pecho (DAP) con cinco métodos diferentes de cálculo entre ellos Smalian y Newton, en donde describe que si el fuste tiene la forma paraboloide con tendencia a cónica el cálculo por la fórmula de Smalian y Newton son similares [17]. Por su parte, la exactitud en la estimación del volumen real depende más a la cantidad y a la precisión de las mediciones de los diámetros en cada sección que del cálculo empleado en sí [18]. En el subconjunto '2' se ubicó el cálculo del volumen por el testigo el cual emplea el factor de forma de 0,70 establecido por el MAG, con una media de 0,95 $\mathrm{m}^{3}$ como lo muestra la Tabla 3.

Dado que en la prueba de Kruskal-Wallis para el factor de forma Tabla 4, el p-valor es menor que 0,05 se acepta la hipótesis que una o varios factores de forma encontrados en este estudio poseen diferencias entre sus medianas.

El análisis comparativo del factor de forma encontrado por el test de Kruskal-Wallis, siendo similares el factor de forma encontrado por las fórmulas de Smalian y Newton con una mediana de 0,46 y 0,47, respectivamente, estos valores concuerdan con el 
Table 3

Subconjuntos homogéneos para el volumen real estimado por cada método en estudio.

Método de cálculo
Cálculo por F. Smalian
Cálculo por F. Newton
Cálculo por M. gráfico
Testigo
Sig.

Table 4

Prueba de Kruskal-Wallis para los factores de forma encontrados.

\section{Chi-cuadrado}

G.L.

Sig.

Rangos

\begin{tabular}{|c|c|c|c|c|}
\hline & & \multicolumn{3}{|c|}{ Factor de forma } \\
\hline \multicolumn{2}{|l|}{ Chi-cuadrado } & \multicolumn{3}{|c|}{244,472} \\
\hline \multicolumn{2}{|l|}{ G.L. } & \multicolumn{3}{|c|}{3} \\
\hline \multicolumn{2}{|l|}{ Sig. } & \multicolumn{3}{|c|}{$0,000^{* * *}$} \\
\hline \multicolumn{5}{|l|}{ Rangos } \\
\hline & Método de cálculo & $\mathbf{N}$ & Rango promedio & Medianas \\
\hline \multirow[t]{5}{*}{ Factor de forma } & F. Smalian & 100 & 119,25 & 0,46 \\
\hline & F. Newton & 100 & 128,14 & 0,47 \\
\hline & M. gráfico & 100 & 212,11 & 0,54 \\
\hline & Testigo & 100 & 342,50 & 0,70 \\
\hline & Total & 400 & & \\
\hline
\end{tabular}

$\mathbf{N}$
100
100
100
100

\begin{tabular}{lc} 
& Subconjunto para alfa $=\mathbf{0 , 0 5}$ \\
\hline 1 & $\mathbf{2}$ \\
0,6311 & \\
0,6336 & \\
0,7210 & 0,9517 \\
& 1,000 \\
\hline 0,657 &
\end{tabular}

estudio donde determina un factor de forma de 0,38 para la especie E. camaldulensis Dehnh, con una muestra de 166 árboles provenientes de una plantación pura a una altitud de 10 a 250 m.s.n.m., los valores de los métodos Smalian y Newton están dentro de un rango aceptable de 0,36 a 0,52. Mientras que el factor de forma encontrado por el método gráfico con una mediana de 0,54 se encuentra fuera del rango permitido, el testigo difiere de las tres medianas mencionadas y fuera del rango permitido con una mediana de 0,70 como se muestra en el Gráfico $1[19,20]$.

\subsection{Validación del volumen calculado con los factores de forma estimados mediante ecuaciones de volumen de una y doble entrada para la especie en estudio}

Se estudian cinco tipos de ecuaciones para volumen con los coeficientes de correlación lineal de Pearson (R) más altos, las variables aplicadas en esta investigación fueron diámetro a la altura del pecho, área basal, altura y volumen comercial calculado por la fórmula de cubicación Smalian de los árboles en estudio (Tabla 5).

Los coeficientes de correlación $(R)$ más altos se dan en las 5 ecuaciones mostradas en la Tabla 6. 


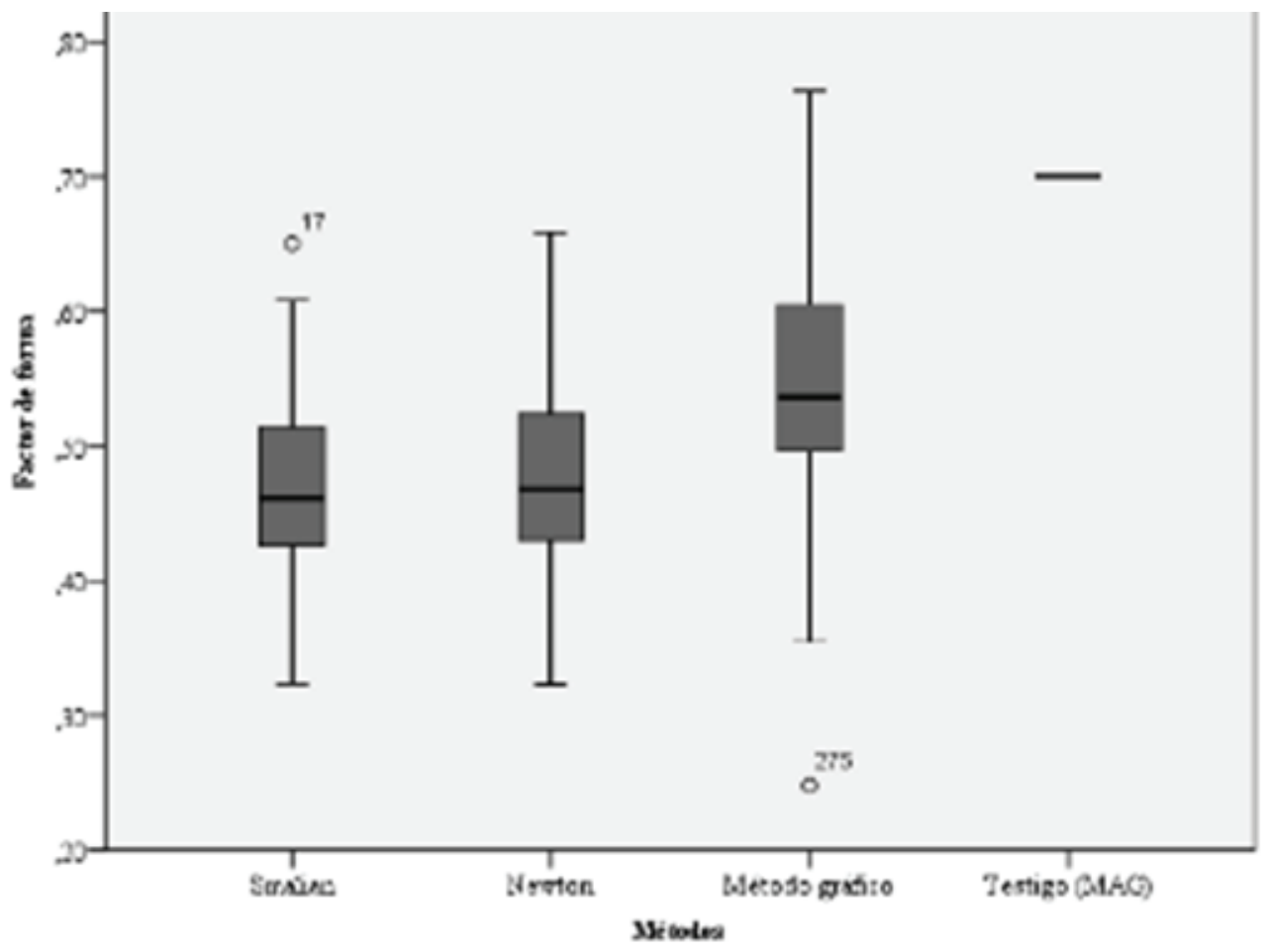

Figure 1

Medianas de los factores de forma encontrados.

Table 5

Coeficientes de correlación de las variables en estudio.

\begin{tabular}{|c|c|c|c|c|c|c|c|c|c|c|c|c|}
\hline & VOL_IT & DAP & H total & $A B$ & $\mathrm{DAP}^{2}$ & $\mathbf{D A P}^{3}$ & 1/DAP & $\mathbf{H}^{2}$ & $H^{*}$ DAP & $\mathrm{H}^{*} \mathrm{DAP} \mathbf{P}^{2}$ & LN_VOL. IT & LN_H*DAP ${ }^{2}$ \\
\hline VOL_IT & 1,00 & & & & & & & & & & & \\
\hline DAP & 0,95 & 1,00 & & & & & & & & & & \\
\hline H total & 0,85 & 0,85 & 1,00 & & & & & & & & & \\
\hline AB & 0,97 & 0,99 & 0,81 & 1,00 & & & & & & & & \\
\hline DAP $^{2}$ & 0,97 & 0,99 & 0,81 & 1,00 & 1,00 & & & & & & & \\
\hline DAP $^{3}$ & 0,96 & 0,95 & 0,75 & 0,99 & 0,99 & 1,00 & & & & & & \\
\hline 1/DAP & $-0,82$ & $-0,94$ & $-0,85$ & $-0,87$ & $-0,87$ & $-0,80$ & 1,00 & & & & & \\
\hline $\mathrm{H}^{2}$ & 0,89 & 0,85 & 0,99 & 0,83 & 0,83 & 0,79 & $-0,82$ & 1,00 & & & & \\
\hline H*DAP & 0,97 & 0,97 & 0,93 & 0,96 & 0,96 & 0,93 & $-0,88$ & 0,95 & 1,00 & & & \\
\hline$H^{*} D A P^{2}$ & 0,99 & 0,96 & 0,85 & 0,98 & 0,98 & 0,98 & $-0,83$ & 0,89 & 0,98 & 1,00 & & \\
\hline LN_VOL. IT & 0,91 & 0,95 & 0,93 & 0,91 & 0,91 & 0,85 & $-0,96$ & 0,92 & 0,95 & 0,90 & 1,00 & \\
\hline LN_H*DAP ${ }^{2}$ & 0,89 & 0,96 & 0,94 & 0,91 & 0,91 & 0,85 & $-0,98$ & 0,92 & 0,96 & 0,90 & 0,99 & 1,00 \\
\hline
\end{tabular}

Se elaboró la regresión lineal por mínimos cuadrados a las cinco ecuaciones propuestas para obtener los valores del coeficiente de determinación. Además, se incluye el análisis de la varianza para determinar la variación de las ecuaciones propuestas como modelos efectivos para fines predictivos; las ecuaciones que no cumplen con los supuestos del modelo de regresión lineal no fueron objeto de estudio.

A partir de los coeficientes encontrados se construyen las ecuaciones de predicción que permite conocer el valor predicho de volumen individual, para cualquier valor de las 
Table 6

Ecuaciones propuestas para el cálculo de volumen.

Número de ecuación
1
2
3
4
5

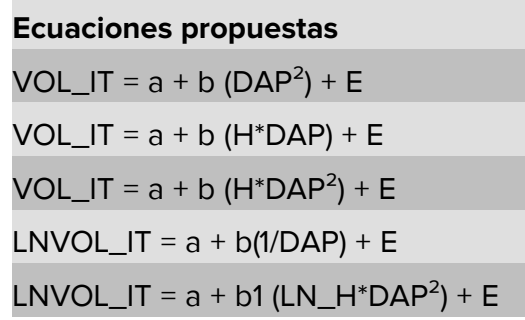

Coeficiente de correlación $\mathbf{R}$
0,97
0,97
0,99
0,96
0,99

variables predictores dentro del dominio de los valores experimentados, los modelos matemáticos potenciales se presentan en la Tabla 7.

\section{Table 7}

Modelos matemáticos de predicción establecidos.

\begin{tabular}{|c|c|c|c|}
\hline Número de ecuación & Ecuaciones propuestas & $\begin{array}{l}\mathbf{R}^{2} \text { coeficiente } \\
\text { determinación }\end{array}$ & Valor crítico de $\mathbf{F}$ \\
\hline 1 & Volumen. Ind = 12,143 (DAP) - 0,1626 & 0,936 & $2,04109 E-60$ \\
\hline 2 & $\begin{array}{l}\text { Volumen. Ind = 0,1399 (H*DAP) - } \\
0,2566\end{array}$ & 0,942 & 2,31374E-62 \\
\hline 4 & $\begin{array}{l}\text { Logaritmo natural (Volumen. Ind) }= \\
-0,5613(1 / \text { DAP })+1,7463\end{array}$ & 0,919 & $2,47629 E-55$ \\
\hline 5 & $\begin{array}{l}\text { Logaritmo natural (Volumen. Ind) }= \\
0,9061\left(\text { logaritmo natural }\left[H^{*} D A P^{2}\right]\right)- \\
0,9647\end{array}$ & 0,978 & 1,04216E-82 \\
\hline
\end{tabular}

Para convalidar el resultado obtenido por las 4 ecuaciones de volumen potenciales con los resultados de cada uno de los cuatro métodos de cubicación Smalian, Newton, método gráfico y testigo se calcula el volumen comercial a partir del diámetro a la altura de pecho con la altura comercial, a partir de las medias de cada método en estudio se calcula el error relativo desde la perspectiva: Tomando los valores de volumen del método de Smalian como el valor exacto y los valores del volumen de las Ecuaciones 1, 2, 4, 5, Newton, método gráfico y testigo como aproximación (Tabla 8).

\section{Table 8}

Valores estimados del volumen comercial por los métodos en estudio.

\begin{tabular}{l|l|l|l|l|l|l|l}
$\begin{array}{l}\text { Volumen. } \\
\text { Smalian }\end{array}$ & $\begin{array}{l}\text { Volumen. } \\
\text { Newton }\end{array}$ & $\begin{array}{l}\text { Volumen } \\
\text { Gráfico }\end{array}$ & $\begin{array}{l}\text { Volumen } \\
\text { Testigo }\end{array}$ & Ecuación $\mathbf{1}$ & Ecuación $\mathbf{2}$ & Ecuación $\mathbf{4}$ & Ecuación $\mathbf{5}$ \\
\hline 0,645 & 0,659 & 0,758 & 0,982 & 0,634 & 0,637 & 0,607 & 0,630
\end{tabular}

Se observa que con el testigo obtiene el valor de mayor error relativo que sobreestima el volumen comercial en un $34,3 \%$, seguido del método gráfico que sobreestima en un $14,8 \%$ el volumen comercial, la Ecuación 4 en cambio subestima el volumen comercial en un $6,2 \%$, mientras que los otros métodos su tasa de subestimación y sobrestimación no supera el $2,4 \%$. En contraste con el estudio realizado en donde la 
Table 9

Cálculo de los errores relativos.

\begin{tabular}{l|l|l|l} 
Método & Valor exacto & Valor de aproximación & Error relativo \\
\hline Volumen Newton & 0,645 & 0,659 & $-2,1 \%$ \\
\hline Volumen Gráfico & 0,645 & 0,758 & $-14,8 \%$ \\
\hline Volumen Testigo & 0,645 & 0,982 & $-34,3 \%$ \\
\hline Ecuación 1 & 0,645 & 0,637 & $1,8 \%$ \\
\hline Ecuación 2 & 0,645 & 0,637 & $1,3 \%$ \\
\hline Ecuación 4 & 0,645 & 0,607 & $6,2 \%$ \\
\hline Ecuación 5 & 0,645 & 0,630 & $2,4 \%$
\end{tabular}

ecuación con la que se estima el volumen de la madera del árbol muestra una mejora de los métodos tradicionales de evaluarlo mediante trozas además muestra que no es tan trascendental determinar que método es el mejor para el cálculo del volumen porque la exactitud en la estimación depende más de las mediciones del diámetro que del cálculo empleado en sí, pero para poder establecer las alturas correspondientes a diámetros determinados o evaluar los diámetros a ciertas alturas, el método polinomial muestra su bondad al tener que resolver una simple ecuación [17].

\section{Conclusiones}

Se determinó el factor de forma de una muestra de 100 individuos con fustes rectos y sin bifurcaciones de la especie $E$. saligna (Eucalipto) en una plantación forestal comercial del sector Tambillo bajo, cantón Colta, provincia de Chimborazo mediante las fórmulas del cálculo del volumen real de Smalian, Newton y método gráfico cuyos valores son de 0,$46 ; 0,47 ; 0,54$, respectivamente.

El análisis comparativo del cálculo de volumen real por secciones la especie en estudio presentó una forma de tipo paraboloide con tendencia de forma cónica, donde el volumen comercial por los métodos Smalian, Newton y método gráfico se encuentran dentro de un mismo grupo homogéneo mientras que el método de cálculo del volumen comercial con el factor de forma de 0,70 se encuentra fuera de este subconjunto homogéneo.

En la verificación del volumen comercial calculado con los factores de forma estimados frente a las ecuaciones de volumen, el error relativo muestra que el método de cálculo del volumen comercial con el factor de forma de 0,70 sobre estima el volumen comercial en un $34,3 \%$, seguido del método gráfico que sobreestima en un $14,8 \%$ el volumen comercial, la Ecuación 4 en cambio subestima el volumen comercial en un $6,2 \%$, mientras que los otros métodos su tasa de subestimación y sobreestimación no supera el 2,4\% además se encontró que la Ecuación 3 no cumple con la condición de normalidad dentro de la regresión lineal por tanto no puede ser aplicada para el cálculo del volumen comercial de la especie en estudio. 


\section{References}

[1] Aguilar C, Sequeira A, Peralta E. Factor de forma para la Tectona grandis L.F [Form factor for Tectona grandis L.F]. Empresa MLR-Forestal. Agrociencia. Siuna, Costa Caribe Norte de Nicaragua. 2016; 50(1):89-105. Spanish

[2] Dirección de Producción Forestal. Manual "Determinación de factores de forma de especies forestales comerciales" [Manual "Determination of commercial forest species form factors"].Ecuador: Ministerio de Agricultura; 2014. p 1-9. Spanish

[3] Rondeux J. Medición de árboles y masas forestales [Measurement of trees and forest stands]. España: Mundi-Prensa. 2010. Spanish

[4] Fontalvo T, De la Hoz Granadillo E, Vergara J. C. Aplicación de análisis discriminante para evaluar el mejoramiento de los indicadores financieros en las empresas del sector alimento de Barranquilla Colombia [Application of discriminant analysis to evaluate the improvement of the financial indicators in the companies of the food sector of Barranquilla - Colombia]. Ingeniare. Revista Chilena de ingeniería. 2012;20(3): 320-330. Spanish

[5] Nájera JA, Hernández HE. Relaciones morfométricas de un bosque coetáneo de la región del Salto, Durango [Morphometric relationships of a contemporary forest in the Salto region, Durango]. Ra Ximhai. 2008;4 (1): 69-81. Spanish

[6] Instituto Nacional de Meteorología e Hidrología (INAMHI). 2013. Memoria técnica: cantón Colta. clima e hidrología. Riobamba - Ecuador: Ministerio de Agricultura (MAG); Coordinación General del Sistema de Información Nacional (CGSIN).

[7] Ministerio de Ambiente del Ecuador. Sistema de clasificación de los Ecosistemas del Ecuador Continental [Classification system of the Ecosystems of Continental Ecuador]. Ecuador: Subsecretaría de Patrimonio Natural. 2012. p 33-35. Spanish

[8] Calvache J. La investigación científica como alternativa en la formación profesional [Scientific research as an alternative in professional training]. Colombia: CEPUN; 2016. Spanish

[9] ETIFN. Procedimientos para la planificación, medición y registro de información del Inventario Forestal Nacional de Paraguay [Procedures for planning, measuring and recording information from the National Forest Inventory of Paraguay]. Manual de Campo. FAO-PNUDPNUMAINFONA-SEAM-FAPI. 2014. Spanish

[10] Pacheco J. Gestión de datos gráficos y tablas dinámicas con excel [Management of graphical data and pivot tables with excel]. Ecuador: Macro. 2015. Spanish

[11] Riecke F. Über die Berechnung des körperlichen [About the calculation of the physical]. Stuttgart: Metzler. 1940. p 75. German

[12] Ecuador Forestal. 2014 Julio [acceso 22 octubre 2018]. Disponible en: http://ecuadorforestal.org/actu alidad-forestal/nuevo-decreto-ejecutivo-no-286-sobre-transferencia-de-competencias-del-ministeri o-del-ambiente-al-magap-responde-a-una-lucha-de-mas-de-15-anos-por-parte-del-sector-forestal/

[13] Nel L. Estadística con SPSS 22 [Statistics with SPSS 22]. Lima: Macro. 2014. Spanish

[14] Morás G. Tablas de volumen de Eucalyptus globulus ssp. globulus al sur del río Negro, Montevideo [Volume tables of Eucalyptus globulus ssp. globulus south of the Negro river, Montevideo,tesis de maestría]. Uruguay: Universidad de la República, Facultad de Agronomía; 2010. Spanish

[15] López C. Conceptos básicos del analisis de regresión [Basics of analysis regression]. Relación alturasdiámetros en las masas. Madrid: Universidad Politécnica de Madrid; 2008. Spanish

[16] Rodríguez H, Ramírez H. Dendrometria Medición forestal [Dendrometria Forest measurement] ResearchGate; 2016. Spanish

[17] Riaño O, Lizarazo I. Estimación del volumen de madera en árboles mediante polinomio único de ahusamiento [Estimation of the volume of wood in trees using a single taper polynomial]. Bogotá: Colombia Forestal. 2017; 20(1): 55-62. Spanish

[18] FAO. El eucalipto en la repoblacion forestal [Eucalyptus in reforestation]. Roma: coleccion FAO Montes. 1981. p 85-96. Spanish

[19] Martin A. Modelos de estimación del volumen de Eucalyptus camadulensis Dehnh, en plantaciones puras en el Ingenio San Antonio, Chinandengua [Models for estimating the volume of Eucalyptus camadulensis Dehnh, in pure plantations at Ingenio San Antonio, Chinandengua,Tesis de grado]. Montevideo, Managua, Nicaragua: Universidad Nacional Agraria; 1997. Spanish

[20] Caillez F. Estimación del volumen forestal y predicción del rendimieno [Forest volume estimation and yield prediction]. Montes: 1980; 22(1):92. 